Submitted to Polymer (an article)

$$
\begin{aligned}
& 1^{\text {st }} \text { submission on } 1 / 7 / 09 \\
& 2^{\text {nd }} \text { submission on } 5 / 7 / 09
\end{aligned}
$$

\title{
Synthesis and Properties of Highly Hydrophilic Polyurethane Based on Diisocyanate with Ether Group
}

\author{
Ken Kojio $^{1, *}$, Yoshitaka Mitsui ${ }^{2}$, and Mutsuhisa Furukawa ${ }^{2}$ \\ ${ }^{1}$ Materials Science and Engineering Department, Faculty of Engineering, \\ ${ }^{2}$ Materials Science Department, Graduate School of Science and Technology, \\ Nagasaki University \\ 1-14 Bunkyo-machi, Nagasaki 852-8521, Japan
}

\footnotetext{
*Authors to whom correspondence should be addressed.

Email: kojio@nagasaki-u.ac.jp
} 


\begin{abstract}
Highly hydropihilic polyurethane elastomers (PUEs) were synthesized from 1,2-bis(isocyanate) ethoxyethane (TEGDI), poly(ethylene oxide-co-propylene oxide) copolyol (EOPO) and 1,4-butane diol/1,1,1-trimethylol propane (75/25)(wt/wt) by a prepolymer method. 4,4'-diphenylmethane diisocyanate (MDI)-based PUEs were synthesized as a control as well. Fourier-transform infrared spectroscopy (FT-IR) and differential scanning calorimetry (DSC) measurement revealed that the degree of microphase separation of the TEGDI-based PUEs was much weaker than for the MDI-based PUEs. Young's modulus and elongation at break of the TEGDI-based PUE were quite lower and larger than for the MDI-based PUEs, respectively. This is due to quite weak cohesion force of the hard segment chains in the TEGDI-based PUEs. The degree of swelling of the TEGDI-based PUEs was five times larger than for the MDI-based one. This is associated with the hydrophilic nature of TEGDI and weak cohesion force in the TEGDI-based PUEs.
\end{abstract}




\section{Introduction}

Polyurethanes (PUs) have been applied for various materials by controlling chemical structures, molecular weight of polymer glycol, hard segment contents, synthesis method, thermal history and so on. When the hydrophilicity is given to the PUs, they can be used as biomaterials[1-4], filter membranes[5], biosensor[6,7], water permeable materials[9], grout materials,[10] etc.

To obtain the hydrophilic PUs, a hydrophilic polymer glycol, for example, poly(oxyethylene) glycol, and a hydrophilic chain extender bearing specific functional groups such as sulfonic acid [11] and quaternary ammonium groups [12] are generally incorporated. If we need to create the PU with a high water absorbing property, it is desirable that all the polymer chains are hydrophilic. However, diisocyanates, for instance, 4,4'-diphenylmethane diisocyanate (MDI) and tolylene diisocyanate (TDI), which have been widely used for the synthesis of PUs, are all hydrophobic. Therefore, the hydrophilic PUs, so far, must absolutely possess a hydrophobic part in the molecular structure.

We investigated the molecular aggregation structure and physical properties of the PUs incorporating 1,2-bis(isocyanate)ethoxyethane (TEGDI). It was revealed that the incorporation of TEGDI induces a phase mixing trend, resulting in the highly softened PU elastomers[13,14] This diisocyanate, TEGDI have another feature, that is, it is comprised of hydrophilic ether groups between diisocyanate groups. Thus, it is expected that we can obtain the PU with a quite high water absorbing property because the incorporation of TEGDI will give a novel network structure, in which the entire chains are hydrophilic.

In this study, hydrophilic polyurethane elastomers (PUEs) were synthesized 
using TEGDI as a diisocyanate and poly(ethylene oxide-co-propylene oxide) copolymer glycol (EOPO) as a soft segment component. The microphase-separated structure, mechanical property and hydrophilicity of the PUEs were investigated.

\section{Experimental section}

TEGDI and MDI were kindly supplied from Nippon Polyurethane Industry, Co., Ltd., Japan. The purities of the TEGDI were greater than 99\%. These diisocyanates were employed as received. EOPO was also kindly supplied from Asahi Glass Urethane, Co., Ltd., Japan. The EO content of EOPO is $80 \mathrm{~mol} \%$ and EO and PO were randomly copolymerized.

PUEs were synthesized by a prepolymer method. The EOPO was dried with bubbling dried nitrogen under a reduced pressure. The residual water content was less than $0.01 \mathrm{wt} \%$, which was obtained by the Karl Fischer titration. TEGDI or MDI was added into dried EOPO $\left(M_{\mathrm{n}}=1020\right.$, 2020) with formulation ratios of $\mathrm{K}=$ $[\mathrm{NCO}] /[\mathrm{OH}]=2.0 \sim 6.0$. A mixture was agitated at $80{ }^{\circ} \mathrm{C}$ under nitrogen atmosphere. One drop of dibutyl tindilaurylate (DBTL, Wako Chemical Co., Ltd. Japan) was added as a catalyst. The reaction was pursued by an amine equivalent method. After the reaction, a mixture of 1,4-buthanediol (BD) and 1,1,1-trimethylol propane (TMP) (BD/TMP=75/25 wt\%) was added into the prepolymer as a crosslinking agent with a condition of NCO INDEX $=[\mathrm{NCO}]_{\text {pre }} /[\mathrm{OH}]=1.03$, where $[\mathrm{NCO}]_{\text {pre }}$ is the [NCO] in the prepolymer. The viscous product was poured into a mold constructed by a spacer of $2 \mathrm{~mm}$ thickness and two aluminum plates heated at $80{ }^{\circ} \mathrm{C}$. The PUEs were demolded after $2 \mathrm{~h}$ curing, and then, they were post-cured at $60{ }^{\circ} \mathrm{C}$ for $24 \mathrm{~h}$ in air. The hard segment content of all the PUE was ca. $44 \mathrm{wt} \%$. . 
The gel fraction and the degree of swelling of the PUEs were determined from weight after equilibrium swelling with toluene and $\mathrm{N}, \mathrm{N}$-dimethylacetamide (DMA) at $50{ }^{\circ} \mathrm{C}$. The degree of gel fraction, $g$, was defined as $g=W_{\mathrm{b}} / W$, where $W_{\mathrm{b}}$ and $W$ are the weight of dried sample once after swelling with a solvent to the equilibrium state and original weight, respectively. The degree of swelling, $q$, was defined as $q=1+\left\{\left(W_{\mathrm{a}}-W_{\mathrm{b}}\right) / d_{\mathrm{s}} /\left(W_{\mathrm{a}} / d_{\mathrm{p}}\right)\right\}$, where $W_{\mathrm{a}}, d_{\mathrm{s}}$, and $d_{\mathrm{p}}$ are the weight of samples swollen to the equilibrium state, the density of solvent, and density of the PUEs, respectively.

Hardness was determined as the International Rubber Hardness Degree (IRHD) using a durometer with the A scale, which is for rubbers in the normal hardness range. These data were measured in general accord with the procedures described in ISO 7619-1 and JIS K 6253.

Attenuated total reflection Fourier transform infrared spectroscopy (ATR-FT-IR) was carried out to confirm the hydrogen bonding state of the hard segment chains. ATR-FT-IR spectra were obtained with an FTS-3000 EXCALIBUR (Varian Japan Co., Ltd. Japan) equipped with an MCT detector using an ATR cell (MIRacle, PIKE Technologies, Inc. USA). All spectra were collected with 32 scans and at a resolution of $4 \mathrm{~cm}^{-1}$.

DSC thermograms were obtained with a DSC (DSC 8230, Rigaku Denki, Co., Ltd. Japan) in the temperature range from -130 to $250{ }^{\circ} \mathrm{C}$ with a heating rate of $10{ }^{\circ} \mathrm{C} \min ^{-1}$ under nitrogen atmosphere. As prepared samples were simply cooled down around $-140{ }^{\circ} \mathrm{C}$, and then, measurements were started.

Temperature dependence of dynamic viscoelastic properties was measured with a dynamic mechanical analyzer (DMS 6100, Seiko Instruments, Co., Ltd., 
Japan). The size of samples used is $30 \mathrm{~mm} \times 5 \mathrm{~mm} \times 2 \mathrm{~mm}$. Measurements were performed in the temperature range from -150 to $250{ }^{\circ} \mathrm{C}$ with a heating rate of $2{ }^{\circ} \mathrm{C}$ $\min ^{-1}$ with nitrogen atmosphere. Imposed strain and frequency were set to be $0.2 \%$ and $10 \mathrm{~Hz}$, respectively.

Tensile testing was performed with an Instron type tensile tester (AGS 100A, Autograph, Shimadzu, Japan) at $20^{\circ} \mathrm{C}$. The dimension of samples was $100.0 \mathrm{~mm} \times 5.0 \mathrm{~mm} \times 2.0 \mathrm{~mm}$. The initial length and elongation rate were set to be $30 \mathrm{~mm}$ and $10 \mathrm{~mm} \mathrm{~min}^{-1}$, respectively.

\section{Results and discussion}

The PUEs were referenced in this report by EOPO molecular weight and the diisocyanate type, TEGDI or MDI, such as 1000-TE and 1000-MD. Table 1 summarized formulation, density and hardness for the MDI- and TEGDI-based PUEs. Appearances of the TEGDI- and MDI-based PUEs were transparent and milky opaque, respectively. Density did not show any trends for all the PUEs. Hardness of the MDI-based PUEs was ca. 80, which is typical number for the MDI-based PUEs. On the other hand, hardness of the TEGDI-based PUEs was 26-36 and considerably smaller than for the MDI-based ones. The reason for that will be discussed later in detail.

To investigate the hydrogen bonding state of the soft and hard segment chains, FT-IR measurement was performed. Figure 1 (a) and (b) shows ATR-FT-IR spectra of $\mathrm{C}=\mathrm{O}$ and $\mathrm{NH}$ stretching band regions $(v(\mathrm{C}=\mathrm{O})$ and $v(\mathrm{NH}))$ for the TEGDI- and MDI-based PUEs. For the MDI-based PUEs, two peaks were observed at ca. 1705 and $1726 \mathrm{~cm}^{-1}$, which can be assigned to hydrogen bonded 
$v(\mathrm{C}=\mathrm{O})$ and free $v(\mathrm{C}=\mathrm{O})\left(v(\mathrm{C}=\mathrm{O})_{\mathrm{H}-\text { bond }}\right.$ and $\left.v(\mathrm{C}=\mathrm{O})_{\text {free }}\right)$, respectively.[15,16] For the TEGDI-based PUE, $v(\mathrm{C}=\mathrm{O})_{\mathrm{H}-\text { bond }}$ and $v(\mathrm{C}=\mathrm{O})_{\text {free }}$ were observed at ca. 1702 and ca. $1715 \mathrm{~cm}^{-1}$, respectively.[13,14] The intensity ratio of $v(\mathrm{C}=\mathrm{O})_{\mathrm{H} \text {-bond }}$, to $v(\mathrm{C}=\mathrm{O})_{\text {free }}$ increased with increasing EOPO molecular weight for the MDI-based PUEs, whereas that was almost comparable for the TEGDI-based PUEs. On the contrary, the spectra in the $v(\mathrm{NH})$ stretching band region were very consistent with that of $v(\mathrm{C}=\mathrm{O})$. Hydrogen bonded $\mathrm{NH}$ groups with ether oxygen $\left(v(\mathrm{NH})_{\text {ether }}\right)$ and urethane carbonyl groups $\left(v(\mathrm{NH})_{\text {carbonyl }}\right)$ and free one $\left(v\left(\mathrm{NH}_{\text {free }}\right)\right)$ are generally observed at around 3290-3310, 3300-3350 and $3450 \mathrm{~cm}^{-1}$, respectively. All spectra showed both hydrogen bonded and free $v(\mathrm{NH})$ as shown in Figure 1 (b). For the MDI-based PUEs, the peak position of hydrogen boned $v(\mathrm{NH})$ shifted from 3291 to $3316 \mathrm{~cm}^{-1}$ with increasing molecular weight of EOPO. Thus, $\mathrm{NH}$ groups in 1000-MD and 2000-MD mainly form hydrogen bonds with ether oxygen of the soft segment and $\mathrm{C}=\mathrm{O}$ of the urethane bonds, respectively. For the TEGDI-based PUEs, the peak positions of hydrogen bonded $v(\mathrm{NH})$ were observed at even higher wavenumber region. Therefore, it seems reasonable to conclude that the aggregation of the hard segment with the formation of hydrogen bonds progressed well with increasing EOPO molecular weight for the MDI-based PUEs. And the aggregation of the hard segment of the TEGDI-based PUEs did not progressed and no EOPO molecular weight dependence was observed. A shoulder was observed in the $v(\mathrm{NH})$ region, and the position is higher than $3450 \mathrm{~cm}^{-1}$. Thus, it may include not only free $v(\mathrm{NH})$ but another bands. The intensity of the shoulder for the TEGDI-based PUEs was larger than for the MDI-based PUEs. As shown in later discussion, the TEGDI-based PUEs possess a large ability of water absorbing. 
Further, the general hydrophobic PUEs do not exhibit the peak at this region. Therefore, it is likely to consider that this shoulder is assignable to free hydroxyl group stretching $(\mathrm{v}(\mathrm{OH}))$ of water adsorbed in the PUEs.

The microphase-separated state of the PUEs was investigated by DSC measurement. Figure 2 shows DSC thermograms of the TEGDI- and MDI-based PUEs. The glass transition temperatures $\left(T_{\mathrm{g}} \mathrm{s}\right)$ of original EOPO with molecular weights of 1000 and 2000 were -67.6 and $-66.2{ }^{\circ} \mathrm{C}$, respectively (not shown). The $T_{\mathrm{g}} \mathrm{S}$ of the soft segment of 1000-TE, 2000-TE, 1000-MD and 2000-MD were observed at $-43.4,-47.5,-6.2$ and $-27.4{ }^{\circ} \mathrm{C}$, respectively. Also, broad base-line shifts were observed at around $100{ }^{\circ} \mathrm{C}$ for all the PUEs, which might be associated with the glass transition of the hard segment chains. The $T_{\mathrm{g}} \mathrm{s}$ of the soft segment of the TEGDI-based PUEs were much lower than for the MDI-based PUEs. $\Delta T_{\mathrm{g}}$, which is a subtraction between the $T_{\mathrm{g}} \mathrm{s}$ of original EOPO and the soft segment in the PUE, were calculated to be 24.2, 20.1, 61.4 and $40.2{ }^{\circ} \mathrm{C}$ for 1000-TE, 2000-TE, 1000-MD and 2000-MD, respectively. Taking a look the influence of the EOPO molecular weight, $\Delta T_{\mathrm{g}}$ of both the TEGDI- and MDI-based PUEs decreased with increasing molecular weight. Generally speaking, when $\Delta T_{\mathrm{g}}$ exhibits the lower number, the soft segment chains get close to a pure phase because the lower number of $\Delta T_{\mathrm{g}}$ implies the smaller number of interaction between ether oxygen in the soft segment and NH group in the hard segment. That is, hard segment solubilization in the soft segment matrix is weak. In contrast, the large number of $\Delta T_{\mathrm{g}}$ indicates a higher degree of structural heterogeneity in which small bundles of the hard segments are more or less solubilized in the soft segment phase. In addition, the glass transition zone width of the TEGDI-based PUEs was much narrower than for 
the MDI-based PUEs. Therefore, according to general interpretation, it seems likely that these trends might indicate that the degrees of microphase separation of the TEGDI- and MDI-based PUEs are quite strong and weak, respectively. However, the interpretation on the TEGDI-based PUEs does not correspond to the FT-IR results. Furthermore, no melting peak of crystallized hard segment domains was observed. We reported that the PUEs based on poly(oxytetramethylene) glycol (PTMG) and TEGDI exhibited quite low $T_{\mathrm{g}}$, which is almost comparable with original PTMG, and the very sharp glass transition in comparison with another commercial diisocyanate-based PUEs and concluded that the TEGDI-PTMG-based PUEs exhibits a phase mixing trend. [13,14] Thus, there might be specific phenomenon for the chain structure of the TEGDI-based PUEs, which prevents interaction between the hard and soft segments. One possibility is the formation of a helix structure of the TEGDI-based hard segments. Though we do not have a concrete interpretation about that, it will be reported somewhere in the future. Therefore, it seems reasonable to conclude from ATR-FT-IR and DSC that the degree of microphase separation of the TEGDI-based PUEs is quite weak, while that for the MDI-based one is slightly stronger and EOPO molecular weight dependence exists.

Figure 3 shows temperature dependence of storage modulus ( $\left.E^{\prime}\right)$ and loss $\operatorname{tangent}(\tan \delta)$ of the TEGDI- and MDI-based PUEs. For all the PUEs, $\alpha$-relaxation of the soft segment, which is associated with the glass transition, was observed as an abrupt decrease in $E^{\prime}$ and a peak in $\tan \delta$. These temperatures and transition widths almost correspond to the $T_{\mathrm{g}} \mathrm{S}$ obtained by DSC. The magnitude of $E$ ' for the TEGDI-based PUEs in the rubbery plateau region was one decade smaller than for the MDI-based ones. This is due to weak cohesion force and high molecular 
mobility of hard segment chains in the TEGDI-based PUEs. For the MDI-based PUEs, there is EOPO molecular weight dependence on $E$ ' in the rubbery plateau region, the magnitudes of $E^{\prime}$ for 2000-MD was greater than for 1000-MD. This can be attributed that the degree of microphase separation became stronger with increasing EOPO molecular weight, resulting in the formation of hydrogen bonded hard segment domains, which can act as physically crosslinking points. In contrast, for the TEGDI-based PUEs, there was no difference between 1000-TE and 2000-TE of the $E^{\prime}$ value in the rubbery plateau region. This is because the hard segment domains were not formed and molecular aggregation state is almost comparable.

Figure 4 shows stress-strain curves of the TEGDI- and MDI-based PUEs. In Table 2, Young's modulus, tensile strength and strain at break obtained from Figure 4 are listed. Stress for the MDI-based PUEs steeply increased with increasing strain, and broke at low strain. The reason for the properties of the MDI-based PUEs is attributed to the presence of hydrogen bonded hard segment domains and the incorporation of TMP. In contrast, stress for the TEGDI-based PUEs gradually increased with strain and it exhibited larger elongation at break. Thus, in other words, Young's modulus and tensile strength for the MDI-based PUEs were high and strain at break was small, while Young's modulus and tensile strength for the TEGDI-based PUEs were low and strain at break was high. Concerning the TEGDI-based PUEs, cohesion force of the hard segment chains is quite lower than for the MDI-based PUEs and the molecular mobility of the hard segment chain is high. These results obtained by tensile testing correspond well to the discussion on dynamic viscoelastic properties.

Table 3 summarizes results on swelling test using DMA, toluene and water 
for the TEGDI- and MDI-based PUEs. The gel fractions of the MDI-based PUEs were almost $100 \%$ for all the solvents, while those for the TEGDI-based PUEs were slightly low. This is due maybe to existence of an incomplete network structure because of low reactivity of TEGDI compared with MDI. The degrees of swelling in toluene of all the PUEs exhibited similar magnitudes. On the other hand, the degrees of swelling of the TEGDI-based PUEs in DMA were much larger than for the MDI-based PUEs. The reason for that can be attributed to the weak cohesion force of the hard segment in the TEGDI-based PUEs. That is, it is quite hard even for DMA molecules to penetrate into the all MDI-BD based hard segment domains. The degree of swelling of the MDI-based PUEs in water was 1.20, even if the soft segment chains, EOPO are hydrophilic. In contrast, the TEGDI-based PUEs exhibited the quite large degree of swelling for water. The TEGDI-based PUEs possess not only the hydrophilic soft segment chains, but also hydrophilic diisocyanate. Furthermore, the hard segment chains of the TEGDI-based PUEs did not form the crystallized domains, but might solubilize into the soft segment phase. Therefore, it seems reasonable to conclude that the phase mixing trend in the PUEs induced by the incorporation of TEGDI and its hydrophilicity successfully produced the highly hydrophilic PUEs.

\section{Conclusions}

The TEGDI- and MDI-based PUEs incorporating hydrophilic EOPO soft segments were synthesized by a prepolymer method. The degree of microphase separation of these two PUEs obtained was totally different. The TEGDI-based PUEs exhibited a phase mixing trend, in contrast, the degree of microphase 
separation of the MDI-based PUEs was slightly stronger and the EOPO molecular weight dependence exists. Based on these chain structures, TEGDI gave the highly softened PUE. The hydrophilicity of the TEGDI-based PUEs was quite high in comparison with that of the MDI-based ones because TEGDI is hydrophilic and the incorporation of TEGDI induced a phase mixing trend. 


\section{References}

1) Silver, J. H.; Karayianni, E.; Cooper, S. L. J Coll Inter Sci 1996, 178, 219-232.

2) Roh, H. W.; Song, M. J.; Han, D. K.; Lee, D. S.; Ahn, J. H.; Kim, S. C. J Biomater Sci, Polym Ed, 1999, 10, 123-143.

3) Mollica, F.; Ventre, M.; Sarracino, F.; Ambrosio, L.; Nicolais, L. Comp Sci Technol 2006, 66, 92-101.

4) Marconi, W.; Martinelli, A.; Piozzi, A.; Zane, D. Biomaterials 1992, 13, 432-438.

5) Pulat, M.; Akdogan, A. J Appl Polym Sci 2002, 85, 193-198.

6) Eggenstein, C.; Borchardt, M.; Diekmann, C.; Grundig, B.; Dumschat, C.; Cammann, K.; Knoll, M.; Spener, F. Biosensors \& Bioelectronics 1999 14, 33-41.

7) Shin, J. H.; Yoon, S. Y.; Yoon, I. J.; Choi, S. H.; Lee, S. D.; Nam, H.; Cha, G. S. Sensors and Actuators B 1998, 50, 19-26.

8) Petrik, S.; Hadobas, F.; Simek, L.; Bohdanecky, M. J Appl Polym Sci 1993, 47, $677-684$

9) Wang, Z. F.; Wang, B.; Ding, X. M.; Zhang, M.; Liu, L. M.; Qi, N.; Hu, J. L. J Membrane Sci 2004, 241, 355-361.

10) Vipulanandan, C.; Liu, J. Cement Concrete Res. 2005, 35, 1754-1763.

11) Siliver, J. H.; Karajianni, E.; Cooper, S. L. J. Coll Inter Sci 1996, 178, 219-232.

12) Marconi, W.; Martinelli, A.; Piozzi, A.; Zane, D. Biomaterials 1992, 13, 432-438.

13) Kojio, K.; Fukumaru, T.; Furukawa, M. Macromolecules 2004, 37, 3287-3291.

14) Furukawa, M.; Mitsui, Y.; Kojio, K. Polymer 2005, 46, 10817-10822. 
15)Lee, H. S.; Wang, Y. K.; Hsu, S. L. Macromolecules 1987, 20, 2089.

16) Brunette, C. M.; Hsu, S. L.; MacKnight, W. J. Macromolecules 1982, 15, 71. 


\section{Captions of Tables and Figures}

Table 1 Formulation, density and hardness of TEGDI- and MDI-based PUEs.

Table 2 Young's modulus, tensile strength, and strain at break for TEGDIand MDI-based PUEs.

Table 3 Swelling behavior of TEGDI- and MDI-based PUEs.

Figure 1 ATR-FT-IR spectra of TEGDI- and MDI- based PUEs. (a) C=O and (b) NH stretching band regions.

Figure 2 DSC thermograms of TEGDI- and MDI- based PUEs.

Figure 3 Temperature dependence of $E^{\prime}$ and $\tan \delta$ of TEGDI- and MDI-based PUEs.

Figure 4 Stress-strain curves of TEGDI- and MDI-based PUEs. 


\begin{tabular}{lcccc}
\hline Sample & HSC/SSC 1$)$ & $\mathrm{K}^{2)}$ & $\begin{array}{c}\text { Density } \\
{\left[\mathrm{g} / \mathrm{cm}^{3}\right]}\end{array}$ & $\begin{array}{c}\text { Hardness } \\
{[\mathrm{IRHD}]}\end{array}$ \\
\hline 1000-MD & $44.2 / 55.8$ & 2.59 & 1.19 & 74.0 \\
2000-MD & $44.4 / 55.6$ & 4.88 & 1.17 & 83.9 \\
1000-TE & $44.4 / 55.6$ & 3.04 & 1.17 & 26.9 \\
2000-TE & $44.4 / 55.6$ & 5.69 & 1.19 & 36.1 \\
\hline 1) HSC: Hard segment content (wt\%), SSC: Soft segment content (wt\%) \\
2) K=[NCO][OH]
\end{tabular}

Table 1

Kojio et al. 


\begin{tabular}{cccc}
\hline Sample & $\begin{array}{c}\text { Young's } \\
\text { modulus } \\
{[\mathrm{MPa}]}\end{array}$ & $\begin{array}{c}\text { Tensile } \\
\text { strength } \\
{[\mathrm{MPa}]}\end{array}$ & $\begin{array}{c}\text { Strain } \\
\text { at break }\end{array}$ \\
\hline 1000-MD & 4.1 & 8.12 & 1.87 \\
2000-MD & 9.4 & 9.30 & 1.54 \\
1000-TE & 0.8 & 2.61 & 7.05 \\
2000-TE & 1.1 & 2.22 & 3.81 \\
\hline
\end{tabular}

Table 2

Kojio et al. 


\begin{tabular}{ccccccccc}
\hline & \multicolumn{3}{c}{ Gel flaction [\% ] } & & \multicolumn{3}{c}{ Degree of swelling } \\
\cline { 2 - 3 } \cline { 7 - 8 } Sample & DMA & Toluene & Water & & DMA & Toluene & Water \\
\hline 1000-MD & 100 & 100 & 99.8 & & 3.99 & 1.47 & 1.20 \\
2000-MD & 97.0 & 100 & 100 & & 3.89 & 1.58 & 1.41 \\
1000-TE & 88.6 & 94.0 & 89.2 & 9.02 & 2.46 & 6.42 \\
2000-TE & 89.5 & 94.9 & 93.4 & 5.18 & 1.81 & 4.00 \\
\hline
\end{tabular}

Table 3

Kojio et al. 


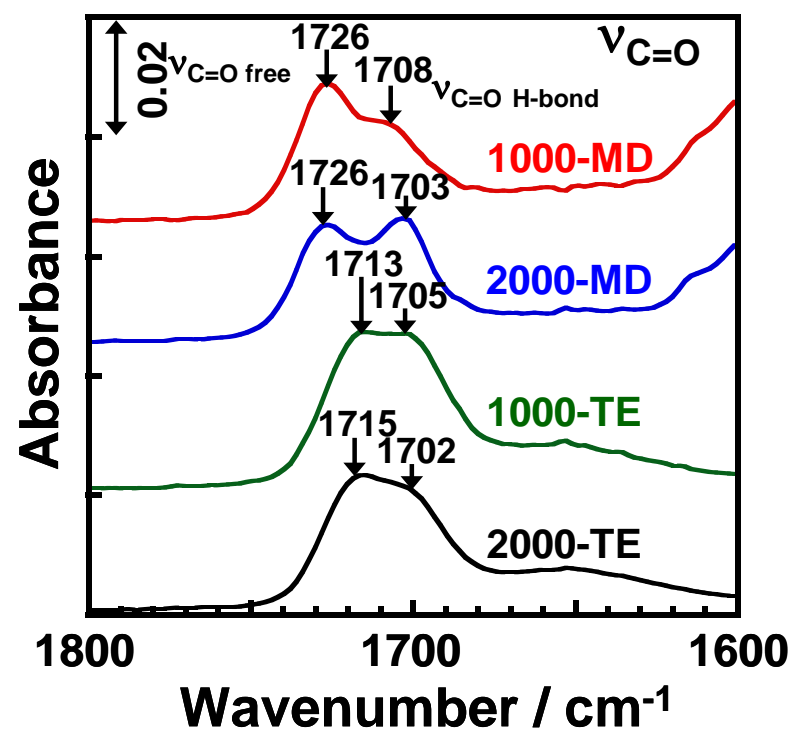

(a)

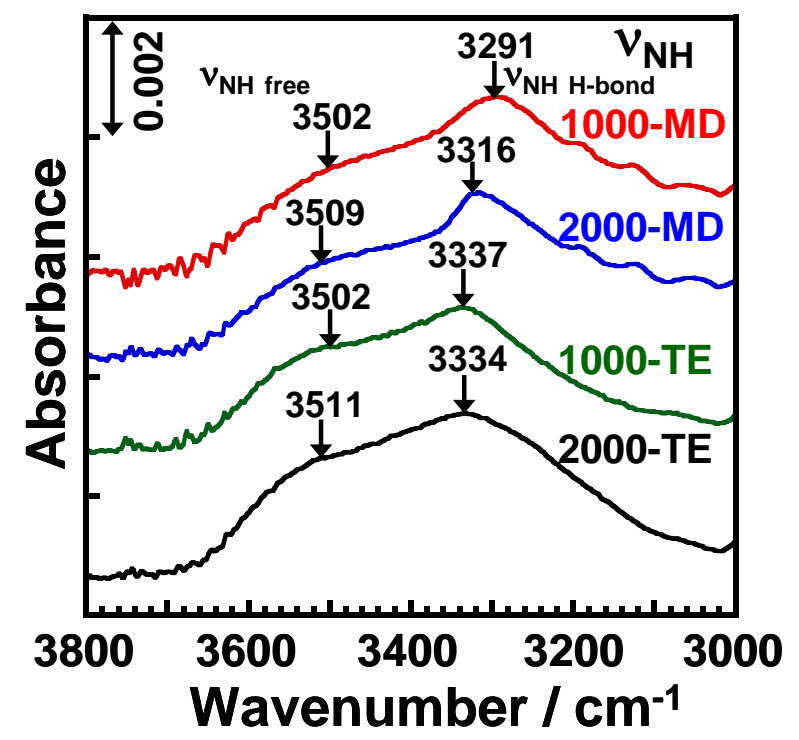

(b)

Figure 1

Kojio et al. 


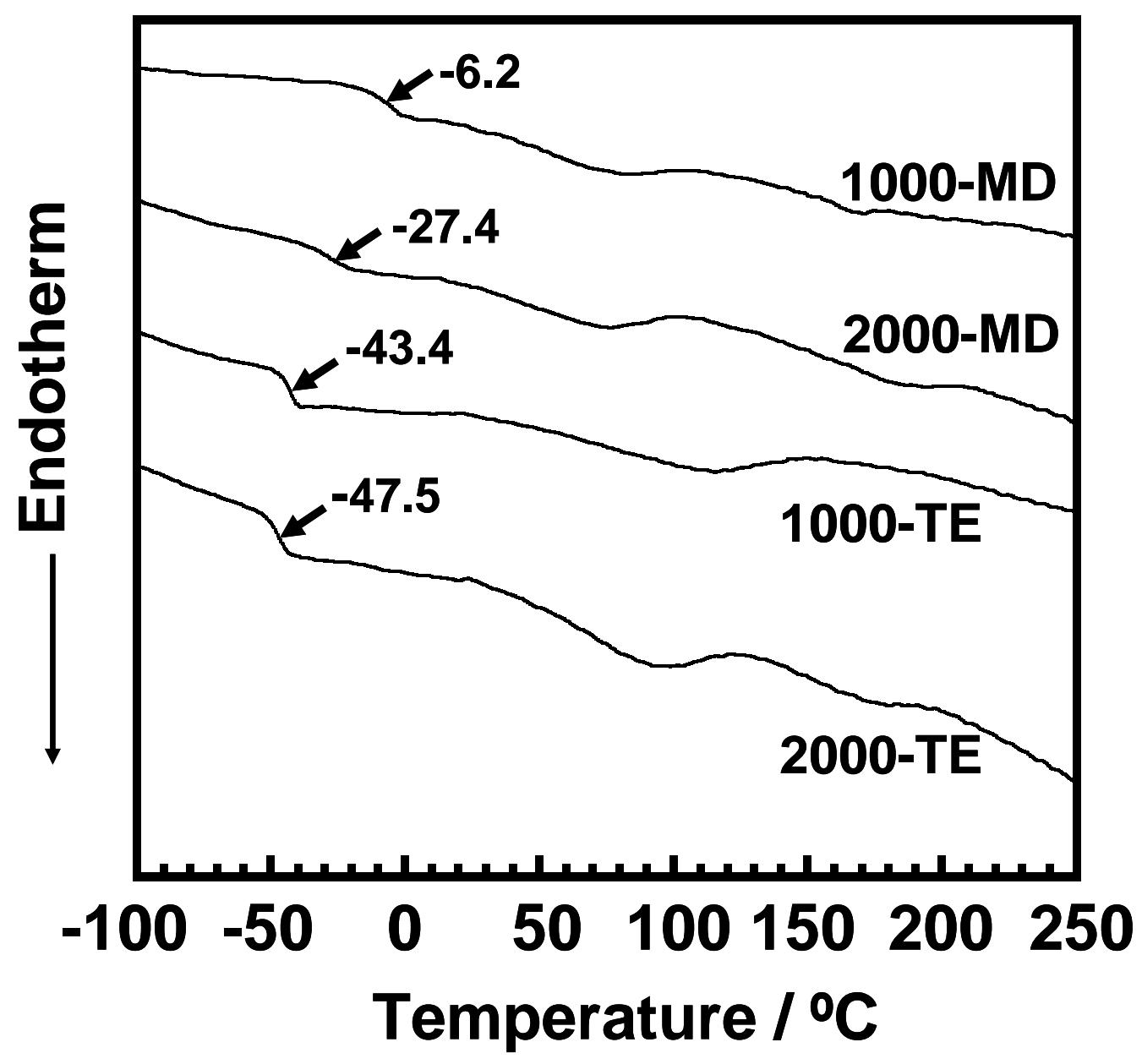

Figure 2

Kojio et al. 


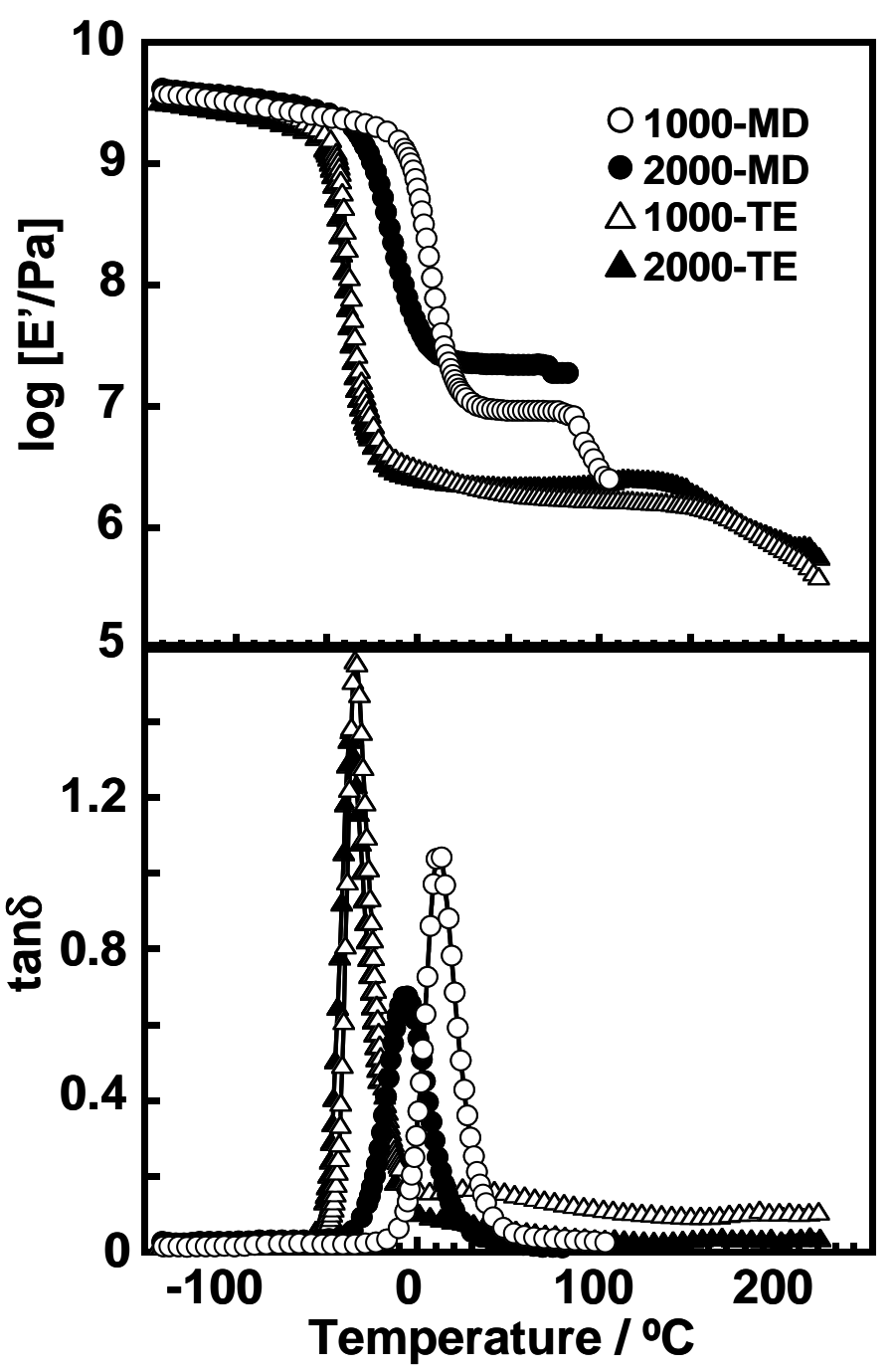

Figure 3

Kojio et al. 


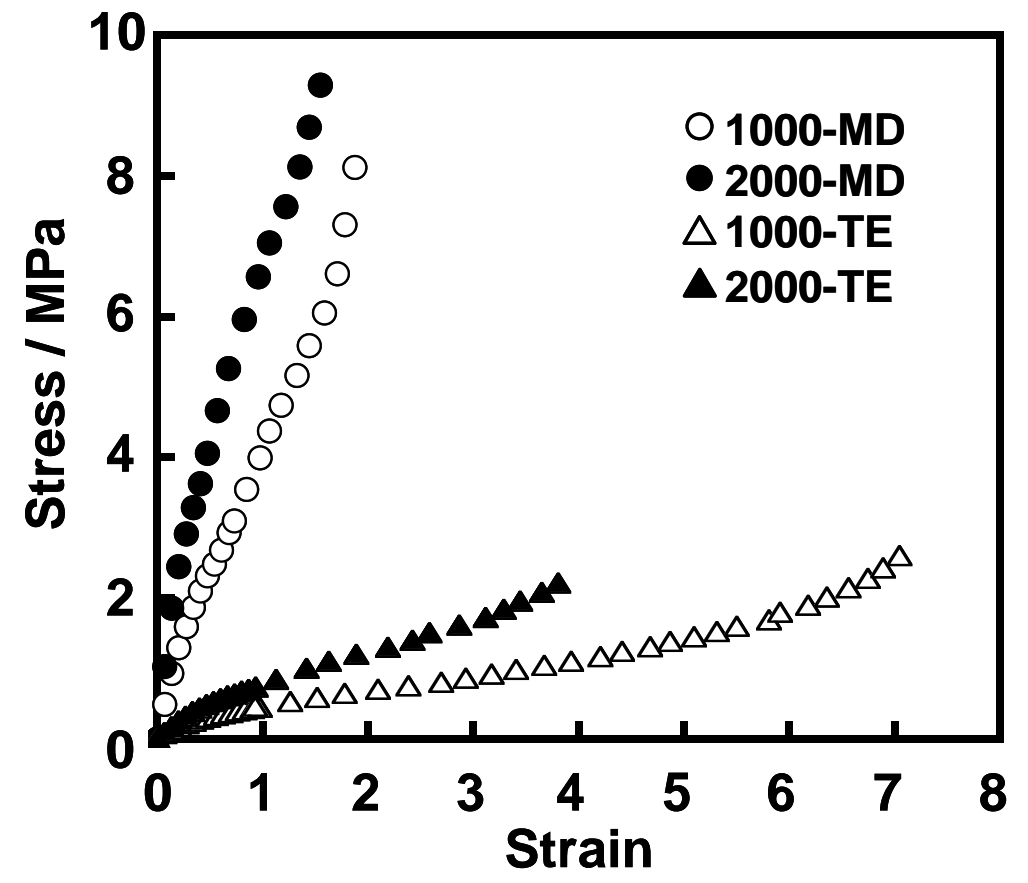

Figure 4

Kojio et al. 